\title{
The absence of intrarenal ACE protects against hypertension
}

\author{
Romer A. Gonzalez-Villalobos, ${ }^{1}$ Tea Janjoulia, ${ }^{1}$ Nicholas K. Fletcher, ${ }^{2}$ Jorge F. Giani, ${ }^{1}$ \\ Mien T.X. Nguyen, ${ }^{2}$ Anne D. Riquier-Brison, ${ }^{3}$ Dale M. Seth, ${ }^{4}$ Sebastien Fuchs, ${ }^{1}$ Dominique Eladari, ${ }^{5}$ \\ Nicolas Picard, ${ }^{5}$ Sebastian Bachmann, ${ }^{6}$ Eric Delpire, ${ }^{7}$ Janos Peti-Peterdi, ${ }^{3}$ L. Gabriel Navar, ${ }^{4}$ \\ Kenneth E. Bernstein, ${ }^{1}$ and Alicia A. McDonough ${ }^{2}$
}

\begin{abstract}
1Departments of Biomedical Sciences and Pathology and Laboratory Medicine, Cedars-Sinai Medical Center, Los Angeles, California, USA. 2Department of Cell and Neurobiology and 3Zilkha Neurogenetic Institute, Keck School of Medicine, University of Southern California, Los Angeles, California, USA. ${ }^{4}$ Department of Physiology and Renal and Hypertension Center of Excellence, Tulane University School of Medicine, New Orleans, Louisiana, USA. INSSERM UMRS 970-Paris Cardiovascular Research Center, Paris, France. ${ }^{6}$ Department of Anatomy, Charite Universitätsmedizin Berlin, Berlin, Germany. 'Department of Anesthesiology, Vanderbilt University School of Medicine, Nashville, Tennessee, USA.
\end{abstract}

\begin{abstract}
Activation of the intrarenal renin-angiotensin system (RAS) can elicit hypertension independently from the systemic RAS. However, the precise mechanisms by which intrarenal Ang II increases blood pressure have never been identified. To this end, we studied the responses of mice specifically lacking kidney angiotensin-converting enzyme (ACE) to experimental hypertension. Here, we show that the absence of kidney ACE substantially blunts the hypertension induced by Ang II infusion (a model of high serum Ang II) or by nitric oxide synthesis inhibition (a model of low serum Ang II). Moreover, the renal responses to high serum Ang II observed in wild-type mice, including intrarenal Ang II accumulation, sodium and water retention, and activation of ion transporters in the loop of Henle (NKCC2) and distal nephron (NCC, ENaC, and pendrin) as well as the transporter activating kinases SPAK and OSR1, were effectively prevented in mice that lack kidney ACE. These findings demonstrate that ACE metabolism plays a fundamental role in the responses of the kidney to hypertensive stimuli. In particular, renal ACE activity is required to increase local Ang II, to stimulate sodium transport in loop of Henle and the distal nephron, and to induce hypertension.
\end{abstract}

\section{Introduction}

Hypertension affects more than 1.5 billion people worldwide and is a key contributor to stroke and cardiovascular and kidney disease. The importance of the renin-angiotensin system (RAS) in the origins of this disorder is underscored by the blood pressure-lowering effects of angiotensin-converting enzyme (ACE) inhibitors and Ang II receptor blockers. However, plasma renin activity, the clinical index used to determine systemic RAS status, is distributed over a wide range in hypertensive subjects $(1,2)$. This observation prompts the suggestion that alterations in tissue-specific RAS, not detected by plasma renin activity, may underlie hypertension. The kidneys play a central role in long-term blood pressure control through their regulation of sodium and fluid balance. Because renal salt retention is strongly influenced by Ang II and there is a complete RAS along the nephron, it has been suggested that increased local Ang II formation may induce hypertension. Indeed, using gene-targeted mice, we and others have shown that increased intrarenal Ang II formation results in hypertension (3-6). As a whole, these observations suggest that renal Ang II synthesis has important consequences for nephron function and the development of hypertension. However, precisely how the intrarenal generation of Ang II elevates blood pressure is not known. Therefore, we tested the hypothesis that, in conditions in which the intrarenal RAS becomes activated, local Ang II synthesis enhances sodium and water reabsorption along the nephron. In addition, we postulated that inhibiting intrarenal Ang II formation effectively protects against hypertension.

Conflict of interest: The authors have declared that no conflict of interest exists. Citation for this article: J Clin Invest. 2013;123(5):2011-2023. doi:10.1172/JCI65460.
Because ACE is responsible for most Ang II synthesis in the kidney (7), we reasoned that the best approach to address our hypothesis was to study the responses of mice with reduced kidney ACE expression to hypertensive stimuli. However, renal ACE is expressed in several cell types, including the proximal tubule, the endothelium of small arterioles, mesangial cells, and distal nephron segments (3). Thus, creating a kidney-specific ACE knockout mouse was impractical. Instead, we studied 2 mouse models in which promoter swapping, via targeted homologous recombination, directed ACE expression to specific cell types while eliminating the enzyme from the kidneys and other tissues. These are ACE 10/10 mice, which express ACE in myelomonocytic cells (8), and ACE $3 / 3$ mice, which express ACE in hepatocytes (9). In ACE $10 / 10$ mice renal ACE activity is totally absent, while in the ACE $3 / 3$ mice it is only $14 \%$ of wild-type values. Moreover, both strains have normal levels of circulating ACE and plasma Ang II. This is important because Ang II availability permits normal kidney development and normal basal blood pressure despite the substantial reduction of kidney $\operatorname{ACE}(8,9)$. Hence, these mice are unique in that they allow us to investigate the role of renal ACE in the setting of normal blood pressure and kidney function.

Here, we report that the absence of renal ACE markedly reduced the hypertension present in response to Ang II infusion (a high serum Ang II model) or reduced nitric oxide production (a low serum Ang II model), 2 experimental conditions in which inflammation and oxidative stress in the renal parenchyma are associated with an abnormal activation of the intrarenal RAS $(10,11)$. Moreover, in the Ang II infusion model, the specific absence of renal ACE reduced local Ang II and the activity of key sodium transporters in the loop of Henle and the distal nephron. This resulted in substantial pro- 

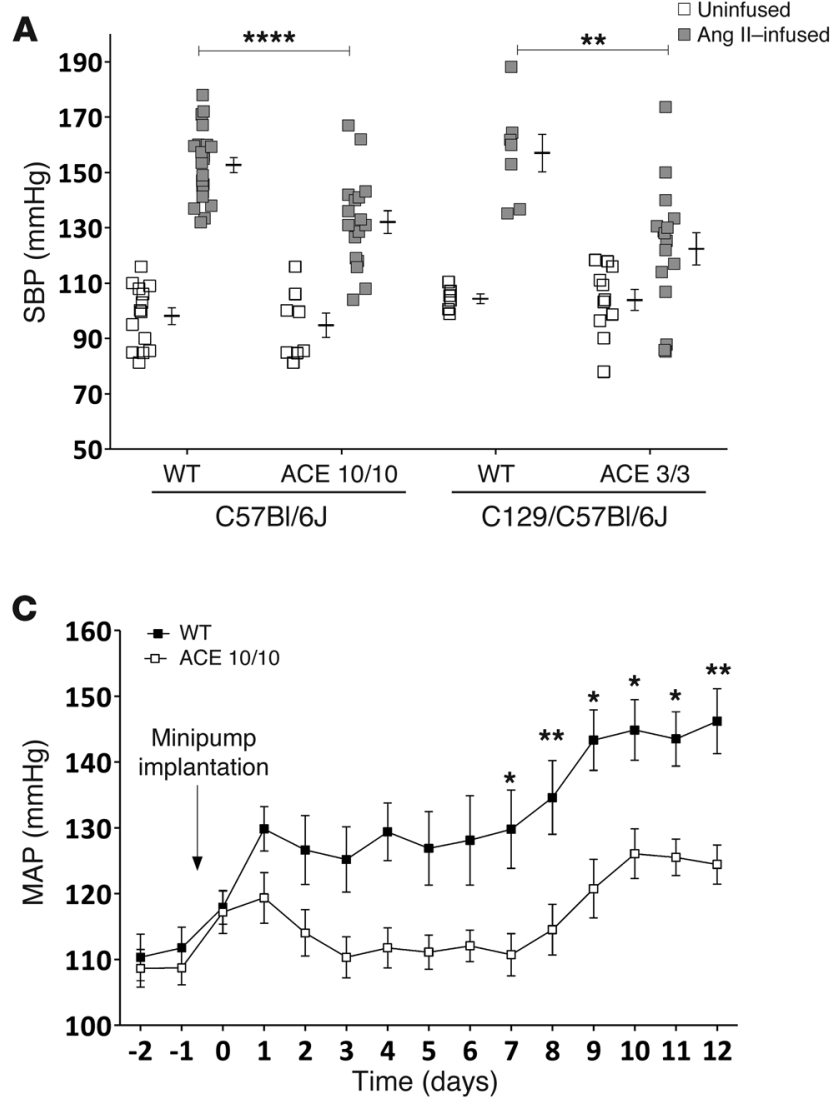

tection against the antinatriuresis and the hypertension induced by systemic Ang II infusion. Thus, even in a model characterized by high serum Ang II, these data implicate the intrarenal generation of Ang II as a critical facilitator of the hypertensive response.

\section{Results}

Mice devoid of kidney ACE are resistant to bypertension. We first investigated whether renal morphology and functionality of the ACE 10/10 mice and ACE 3/3 mice were normal. We did this by histological analysis of kidney tissues (data not shown) and by testing the urine concentration capacity of the mutant mice (Supplemental Figure 1; supplemental material available online with this article; doi:10.1172/JCI65460DS1). These and previous experiments demonstrated that at baseline there is normal histology and renal concentration ability in kidneys from the mutant mice (9). The Ang II-induced hypertension model was used in most of our experiments. Our reasoning was twofold. First, this approach is believed by many to recapitulate several features of human essential hypertension. Second, strong evidence indicates that subpressor doses of Ang II activate the intrarenal RAS and, consequently, induce local Ang II formation (11). The infusion rate of $400 \mathrm{ng} /$ $\mathrm{kg} / \mathrm{min}$ was selected on the basis of previous experiments showing that this dose induces a slowly progressive hypertension in which the Ang II effects of appetite suppression and depressed body weight gain are avoided (Supplemental Figure 2A). In addition, we chose to use only male mice to avoid the confounding effects of sexual dimorphism in hypertension (12).

Under basal conditions, the systolic blood pressure of ACE 10/10 and ACE $3 / 3$ mice, as measured by tail-cuff plethysmography, was

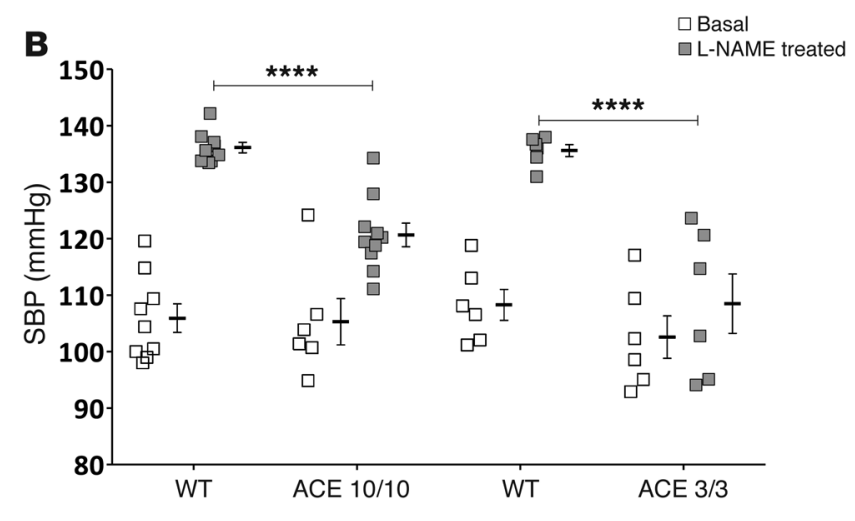

Figure 1

The absence of kidney ACE blunts the hypertensive response to Ang II infusion or L-NAME. (A) Systolic blood pressure (SBP) of wild-type, ACE 10/10, and ACE 3/3 mice after 2 weeks of Ang II infusion (400 $\mathrm{ng} / \mathrm{kg} / \mathrm{min}$ via minipump). Blood pressure was measured by tail-cuff plethysmography. $n=6-22$ per group. The corresponding background strain is indicated. (B) Systolic blood pressure of wild-type, ACE 10/10, and ACE $3 / 3$ mice after 2 weeks of L-NAME treatment $(5 \mathrm{mg} / 10 \mathrm{ml}$ in the drinking water). $n=6-9$ per group. (C) MAP of wild-type and ACE 10/10 mice during chronic Ang II infusion. MAP was recorded by telemetry. $n=7-8$ per group. Uninfused, sham-operated mice; Basal, mice not receiving L-NAME in the drinking water. ${ }^{*} P<0.05$, ${ }^{* \star} P<0.01$, ${ }^{* * * \star} P<0.0001$. Values represent individual mice and mean \pm SEM.

similar to that of wild-type mice of the same genetic background (Figure 1A). However, when subjected to chronic Ang II infusion for 2 weeks, the hypertensive response was blunted in both strains devoid of kidney ACE. Specifically, the blood pressure was 20 $\mathrm{mmHg}$ lower in the ACE 10/10 mice and $47 \mathrm{mmHg}$ lower in the ACE $3 / 3$ mice as compared with that in equally treated wild-type mice. The effects of the lack of intrarenal ACE were also examined in the hypertension induced by $\mathrm{N}_{\omega}$-Nitro-L-arginine methyl ester hydrochloride (L-NAME). Inhibition of nitric oxide synthesis with L-NAME induces a low renin and low plasma Ang II form of hypertension (13). Under these conditions, ACE 10/10 and ACE $3 / 3$ mice still manifested a blunted hypertensive response (Figure $1 \mathrm{~B}$ ).

From this point on, experiments were conducted only in the ACE $10 / 10$ mice, because the effects of experimental hypertension are better characterized in their genetic background strain (C57BL/6J) $(14,15)$. Using telemetry (Figure 1C), we confirmed an equivalent baseline 24-hour mean arterial pressure (MAP) in wild-type and ACE 10/10 mice. Further, in response to chronic Ang II infusion, the rise in ACE 10/10 MAP was 20-26 $\mathrm{mmHg}$ lower than that in wild-type MAP (Figure 1C).

The lack of kidney ACE prevents intrarenal Ang II accumulation and sodium and water retention induced by chronic Ang II infusion. To better understand the effects of intrarenal Ang II on blood pressure, we focused on Ang II-induced hypertension. This model has the advantage that Ang II infusion maintains high serum levels of this peptide, minimizing any effects of plasma Ang II fluctuations. Indeed, our time-based analysis demonstrated that plasma Ang II levels were similar in wild-type mice and ACE 10/10 mice during the infusion (Supplemental Figure 3), with the exception of higher 

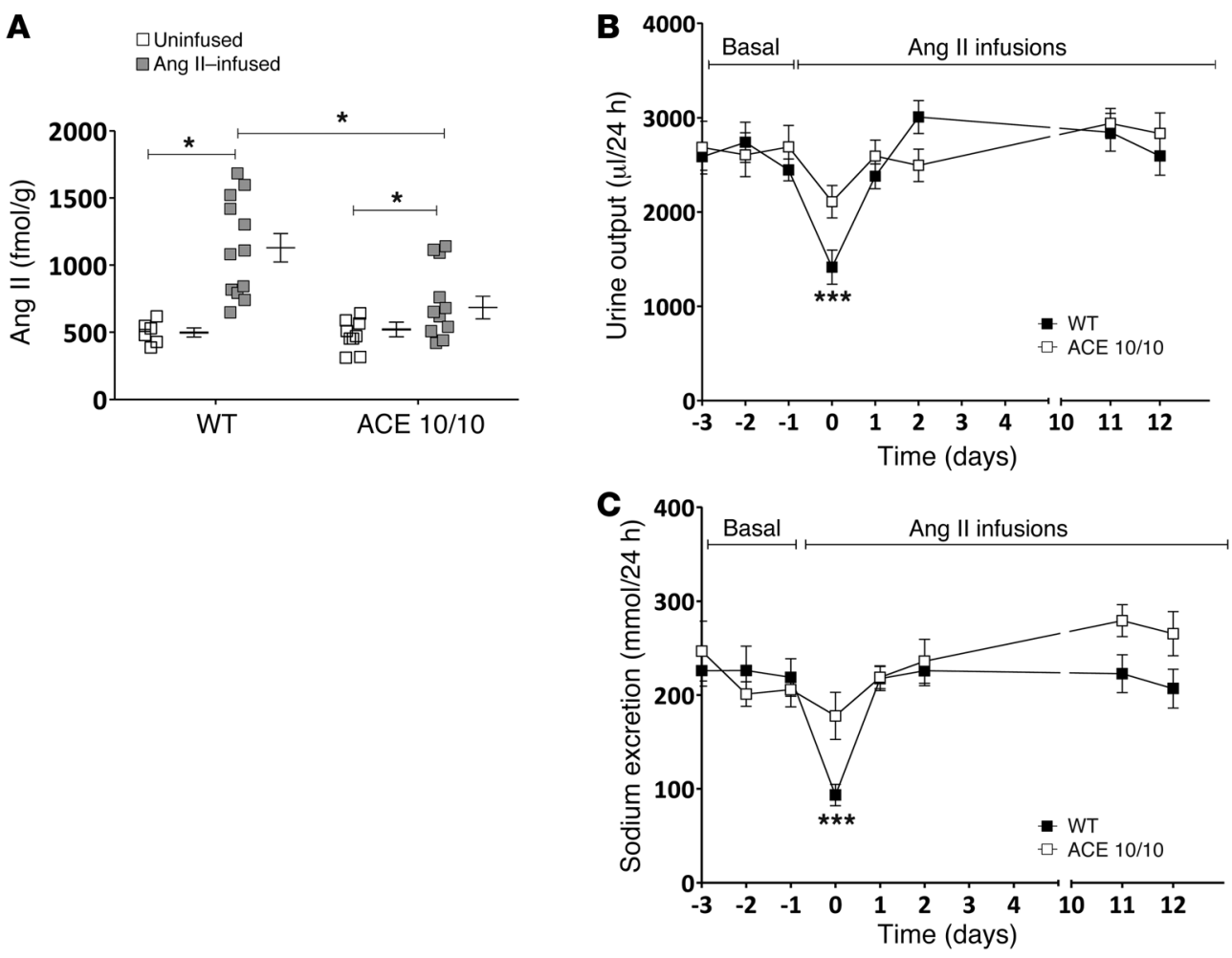

\section{Figure 2}

The absence of kidney ACE reduces both renal Ang II accumulation and sodium and water retention in response to Ang II infusion. (A) Kidney Ang II content of wild-type and ACE 10/10 mice after 2 weeks of Ang II infusion (400 ng/kg/min via minipump). Ang II concentration was measured by radioimmunoanalysis. $n=6-12$ per group. (B) Urine and (C) sodium output of wildtype and ACE 10/10 mice during chronic Ang II infusion. Mice were housed individually in metabolic cages, with free access to food and water. Results are expressed as daily averages. $n=8-10$ per group. ${ }^{\star} P<0.05,{ }^{* *} P<0.001$. Values represent individual measurements and mean \pm SEM.
Ang II levels in wild-type mice after day 1 after implantation. In analyzing the effects of Ang II infusion on the concentrations of this peptide in the kidneys (Figure 2A), we observed a significant increase in Ang II levels in wild-type mice (130\% $\pm 21 \%)$, consistent with previous reports (15). In contrast, ACE 10/10 mice had a significantly lesser increase in intrarenal Ang II ( $45 \% \pm 16 \%)$. To evaluate the consequences of reduced Ang II formation on renal function, ACE 10/10 and wild-type mice were housed individually in metabolic cages to determine urine volume and sodium output. Baseline sodium and urine excretion were similar between wildtype and ACE 10/10 mice (Figure 2, B and C). Within 24 hours after initiating Ang II infusion, wild-type mice displayed a substantial reduction in urine excretion $(43 \% \pm 7 \%$ decrease, $P<0.001)$ and an even greater reduction in sodium excretion $(58 \% \pm 5 \%$, $P<0.001)$. Sodium and urine output returned to basal levels after 48 to 72 hours, coincident with the rise in blood pressure (Figure 1C). Thus, sodium balance was likely reestablished by pressure-natriuresis response. Hereafter, sodium balance was attained at the expense of the hypertension, consistent with a major shift in pressure-natriuresis relationship. In contrast, the initial urine and sodium excretion reductions were not significant in the ACE 10/10 mice $(22 \% \pm 6 \%$ and $22 \% \pm 12 \%$, respectively, NS) and returned to baseline after 48 to 72 hours without coincident increases in blood pressure. Hence, there was an adequate renal compensation without adjustments of the pressure-natriuresis relationship. Finally, these changes cannot be attributed to water and food consumption fluctuations, as they remain stable throughout the experiment (Supplemental Figure 4).

In view of these findings, we studied the expression of RAS components. Recently, it was reported that most angiotensinogen found in the kidney comes from the liver (16). Accordingly, we analyzed the protein expression of liver and kidney angioten- sinogen in tissue homogenates. Ang II infusion increased liver angiotensinogen to a similar extent in wild-type and ACE 10/10 mice (Figure $3 \mathrm{~A}$ ). In contrast, baseline kidney angiotensinogen levels were significantly lower in ACE 10/10 mice as compared with those in wild-type mice. Moreover, kidney angiotensinogen content increased only in wild-type mice in response to Ang II infusion (Figure 3A). Similarly, Ang II increased urinary angiotensinogen excretion only in wild-type mice (Supplemental Figure 5). Because ACE in the ACE 10/10 mice is expressed exclusively in myelomonocytic cells and there was renal macrophage infiltration in both wild-type and mutant mice during Ang II infusion (Supplemental Figure 6), we also determined the expression of kidney ACE (Figure 3B). As expected, baseline kidney ACE expression was negligible in the ACE 10/10 mice. Further, while Ang II infusion caused an increased in ACE expression in wild-type mice, such an effect was not observed in the ACE 10/10 mice. Thus, even in the presence of marked levels of macrophage infiltration, ACE expression remained substantially lower in the ACE 10/10 mice. Finally, the lower renal Ang II levels of ACE 10/10 mice during Ang II infusion cannot be attributed to lower $\mathrm{AT}_{1}$ receptor expression, as this was similar to that observed in hypertensive wild-type mice (Supplemental Figure 7).

A major role of ACE-derived Ang II formation in the activation of renal sodium transporters in the loop of Henle and distal nephron. Because the potent long-term antinatriuretic effects of Ang II are primarily due to increased tubular reabsorption rather than to reductions in glomerular filtration rate $(17,18)$, we hypothesized that Ang IIinfused ACE 10/10 mice, lacking intrarenal ACE and Ang II production, might exhibit a blunted Ang II-dependent activation of renal sodium transporters compared with wild-type mice. Therefore, we profiled the expression of the major sodium and chloride transporters along the nephron. These included the proximal 
A
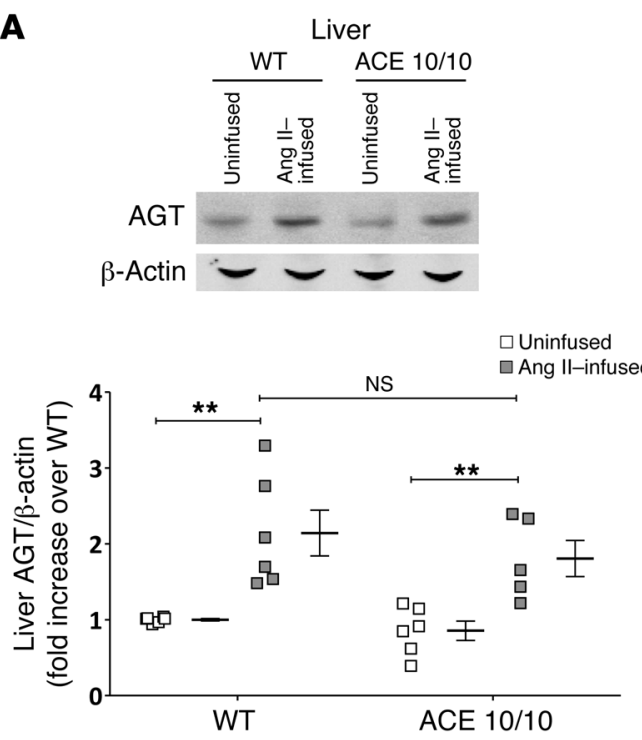

B

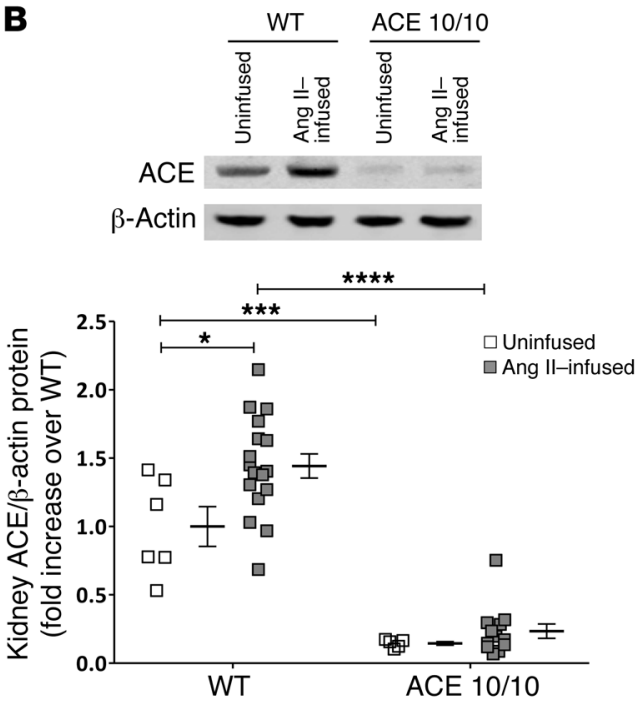

tubule $\mathrm{Na}^{+} / \mathrm{H}^{+}$exchanger 3 (NHE3), the loop of Henle $\mathrm{Na}^{+} / \mathrm{K}^{+} / 2 \mathrm{Cl}^{-}$ cotransporter 2 (NKCC2), the distal tubule $\mathrm{NaCl}$ cotransporter (NCC), the epithelial sodium channel (ENaC), the anion exchangers pendrin and $\mathrm{Na}^{+}$-dependent $\mathrm{HCO}_{3}-\mathrm{Cl}^{-}$exchanger (NDBCE), and the $\mathrm{Na}^{+} / \mathrm{K}^{+}$ATPase. At baseline, transporter levels in wild-type and ACE 10/10 mice were not significantly different (Supplemental Figure 8). A 2-week Ang II infusion into wild-type mice changed the expression levels of several transporters, most prominently, the loop of Henle NKCC2 and the distal tubule NCC (Figure 4). NKCC2 phosphorylation increased 4 fold and the abundance and phosphorylation levels of NCC were 1.6- and 5-fold increased, respectively. More distal transporters also displayed changes associated with activation (Figure 5): the abundance of the $\alpha$ subunit of $\mathrm{ENaC}$ and the cleavage of the $\alpha$ and $\gamma$ subunits, measures of channel activation (19), were 2 -fold increased, and pendrin abundance was also increased by 1.3 fold (Figure 5). The expression of other transporters was unaltered (Supplemental Figure 9). Importantly, ACE 10/10 mice failed to display significant changes in any of the transporters tested in response to Ang II infusion, the excep-
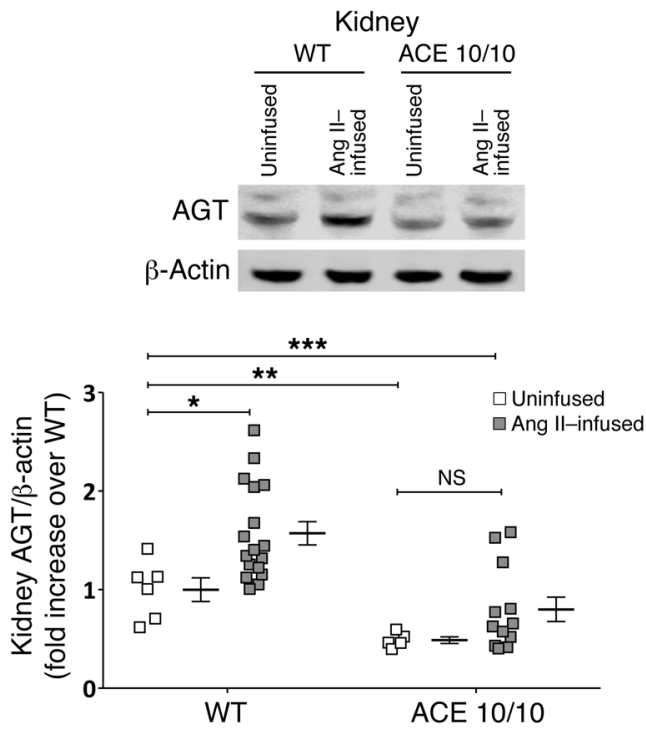

\section{Figure 3}

Changes in liver and renal angiotensinogen and renal ACE protein expression in wild-type and ACE 10/10 mice during Ang II infusion. (A) Angiotensinogen (AGT) and (B) ACE expression were analyzed in total tissue homogenates from wild-type and ACE 10/10 mice 2 weeks after sham operation (uninfused group) or after 2 weeks of Ang II infusion (400 $\mathrm{ng} / \mathrm{kg} / \mathrm{min}$ via minipump). Immunoblots were performed with a constant amount of protein per lane for angiotensinogen, ACE, and $\beta$-actin (Supplemental Table 1). Values represent individual measurements and mean \pm SEM. $n=6-17$ per group. ${ }^{*} P<0.05,{ }^{* \star} P<0.01$, ${ }^{* \star \star} P<0.001,{ }^{* \star \star \star} P<0.0001$.

tion being the cleavage of the $\alpha$ and $\gamma$ subunits of $\mathrm{ENaC}$ (Figure 5 and Supplemental Figure 9).

To better characterize the timing of these changes, we performed transporter profiling at days 1, 4, and 10 after infusion. At day 1 , we did not observed any significant differences in the transporter profile (data not shown). After 4 days of infusion, wild-type mice showed 0.27 -fold increased phosphorylation of NHE3. In contrast, ACE 10/10 mice showed significant decreases in total abundance and phosphorylation of NKCC2 (Supplemental Figure 10). After 10 days, the changes in phosphorylation and abundance in the transporter profile were similar, although of lesser magnitude, to those observed at the end of the experiment. Briefly, in wild-type mice, NHE3 phosphorylation had returned to baseline, NKCC2 phosphorylation was 0.55 -fold increased, and the abundance and phosphorylation of NCC were 0.59 - and 2 -fold increased, respectively.

Extended analysis of NKCC2 and NCC. We assessed the physiological correlates of changes in NKCC2 and NCC during Ang II infusion, the 2 transporters in which we found the largest changes. For this, 
A

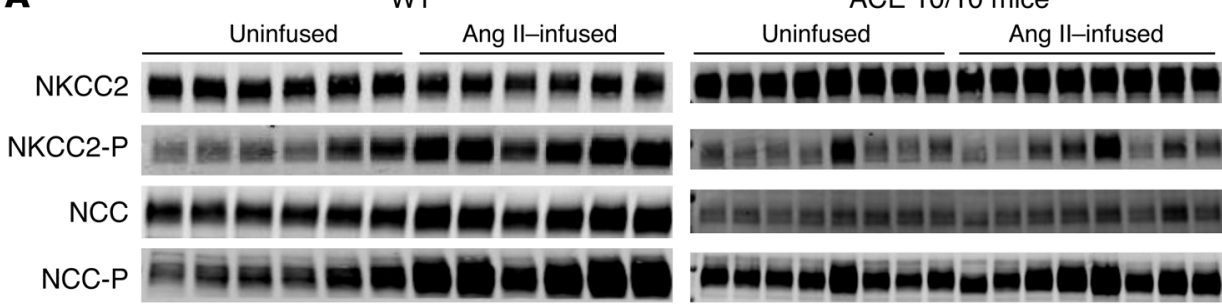

B
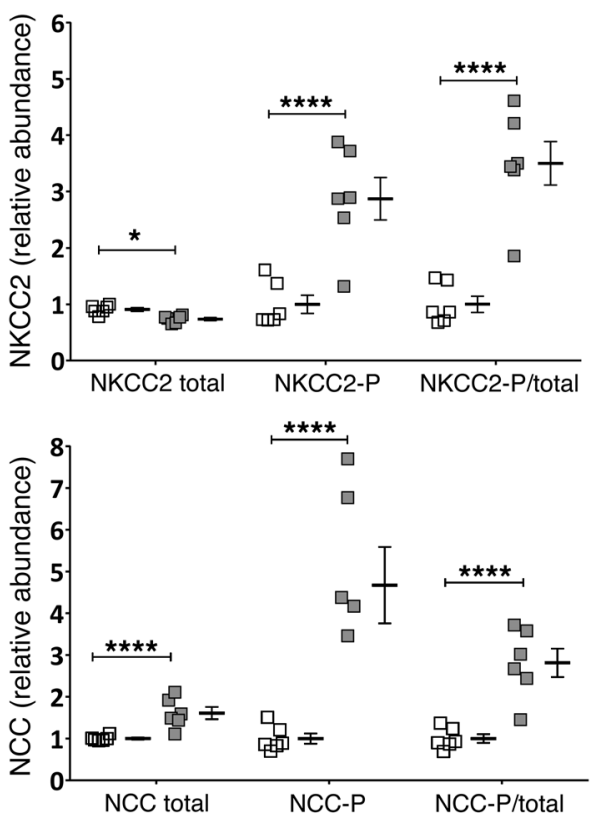
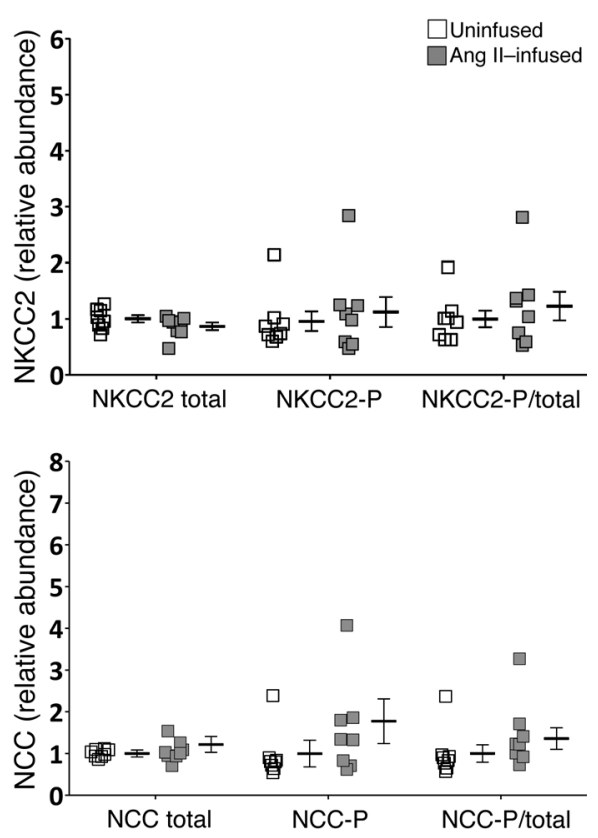

Figure 4

The absence of kidney ACE prevents changes in expression of NKCC2 and NCC induced by Ang II infusion. Transporter expression was analyzed in whole renal tissue homogenates from wild-type and ACE 10/10 mice 2 weeks after sham operation (uninfused group) or after 2 weeks of Ang II infusion (400 $\mathrm{ng} / \mathrm{kg} / \mathrm{min}$ via minipump). (A) Immunoblots were performed with a constant amount of protein per lane (Supplemental Table 1). (B) Relative abundance of total and NKCC2-P and NCC as well as the phosphorylated/total ratios of is displayed as individual measurements and mean \pm SEM. $n=6-8$ per group. ${ }^{*} P<0.05,{ }^{* * *} P<0.0001$. we measured acute natriuretic and diuretic responses (over 3 hours) of wild-type and ACE 10/10 mice to a single bolus of furosemide, a NKCC2 blocker, and hydrochlorothiazide (HCTZ), a NCC blocker (Figure 6A and Supplemental Figure 11). The responses were calculated as the fold change in mice injected with diuretics compared with mice injected with vehicle. At baseline, both strains displayed significant natriuretic responses to furosemide and HCTZ. The natriuretic and diuretic responses to furosemide and HCTZ increased significantly during Ang II infusion in wild-type mice but not in the ACE 10/10 mice. These functional responses paralleled the Ang II-increased abundance of phosphorylated NKCC2 (NKCC2-P), NCC, and phosphorylated NCC (NCC-P) in wild-type mice and the lack of change in these transporters in the ACE 10/10 mice. Phosphorylation of NKCC2 and NCC is associated with their expression in the apical membrane and transporter activation $(20,21)$. We further validated the immunoblot changes by confocal microscopy, which illustrated specific increases in phosphorylation of these transporters in Ang IIinfused wild-type mice but not in ACE 10/10 mice treated in an identical fashion (Figure 6B). Finally, fractionation of membranes to enrich plasma membranes and intracellular membranes (21) revealed that most (>90\%) NKCC2-P and NCC is located in plasma membranes in both strains of mice (Supplemental Figure 12).

The oxidative-stress responsive kinase 1 (OSR1) and the STE20 (Sterile 20)/SPS1-related proline/alanine-rich kinase (SPAK) are 2 kinases with a high degree of structural homology that phosphorylate and activate NKCC2 and NCC (22-24). We did not detect a change in OSR1 abundance in response to Ang II infusion (Supplemental Figure 9). SPAK, whose expression colocalizes in the kidney with thick ascending limb NKCC2 and distal convoluted tubule NCC, exists in multiple isoforms in kidney: a full-length isoform with kinase activity (FL-SPAK); an isoform with a truncated $\mathrm{N}$ terminus (SPAK2), predicted to be kinase defective; and the smaller "kidney-specific" isoform (KS-SPAK), lacking the kinase domain and capable of inhibiting phosphorylation of NKCC2 and NCC by OSR1 and FL-SPAK, respectively (25). To identify the location of the 3 isoforms on immunoblots, we probed whole kidney homogenates with antibodies generated against the $\mathrm{C}$ - and the $\mathrm{N}$-termini (Supplemental Figure 13). In whole kidney homogenates, the relative abundance of these isoforms was FL-SPAK>SPAK2>KS-SPAK (Figure 7A). Baseline levels of SPAK were similar in wild-type and ACE 10/10 mice, while SPAK2 and KS-SPAK were approximately $20 \%$ lower in the ACE 10/10 mice (Supplemental Figure 8). In wild-type mice, a 2-week Ang II infusion had no effect on FL-SPAK or SPAK2 but significantly decreased KS-SPAK and increased the FL-SPAK/ KS-SPAK ratio by more than 50\% (Figure 7A). Consistent with this finding, we observed significant increases in the phosphorylation levels of OSR1 and SPAK only in wild-type kidneys after Ang II infusion (Figure 7B). Furthermore, our time-based analysis indicated that SPAK activation occurred early during the hypertension. Specifically, at day 4 after infusion, we found strong induction of FL-SPAK, the FL-SPAK/KS-SPAK ratio, and phosphorylation of SPAK as well as a reduction of KS-SPAK in 
A

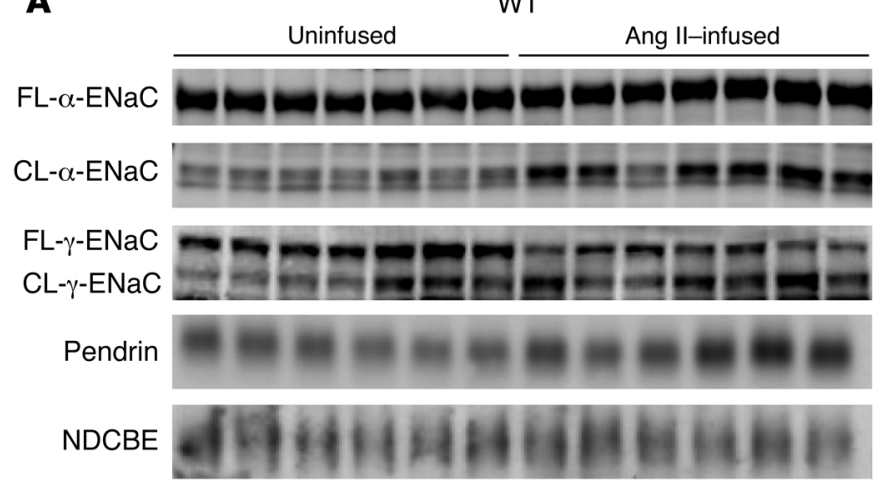

B
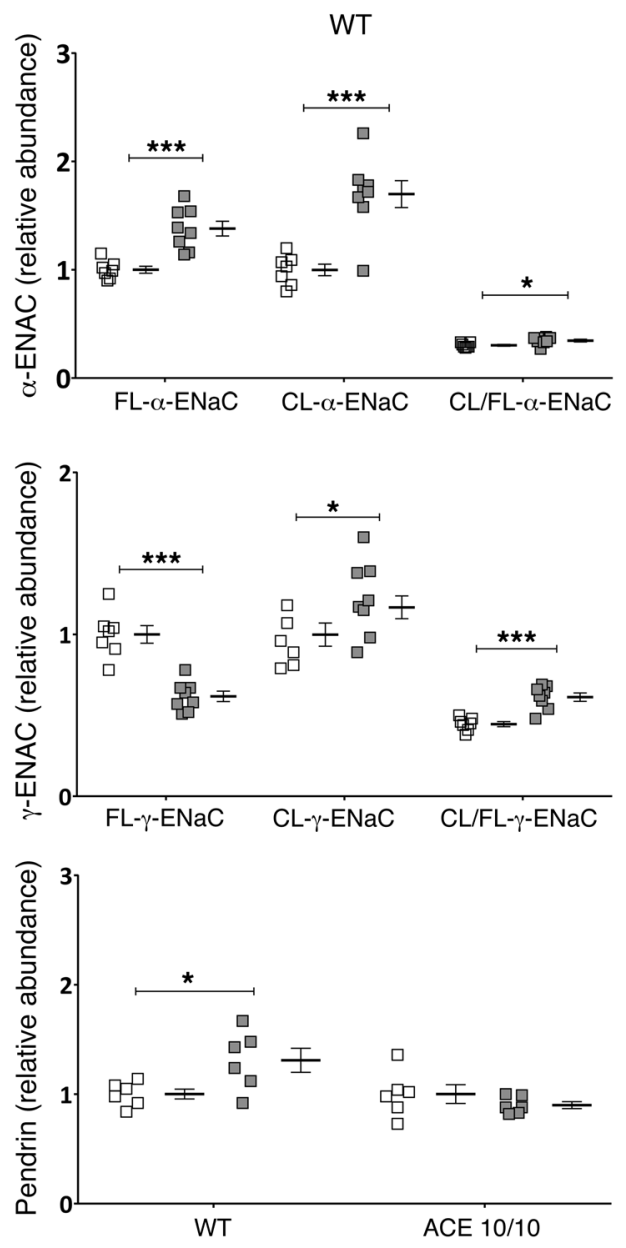

ACE 10/10 mice

Uninfused Ang II-infused
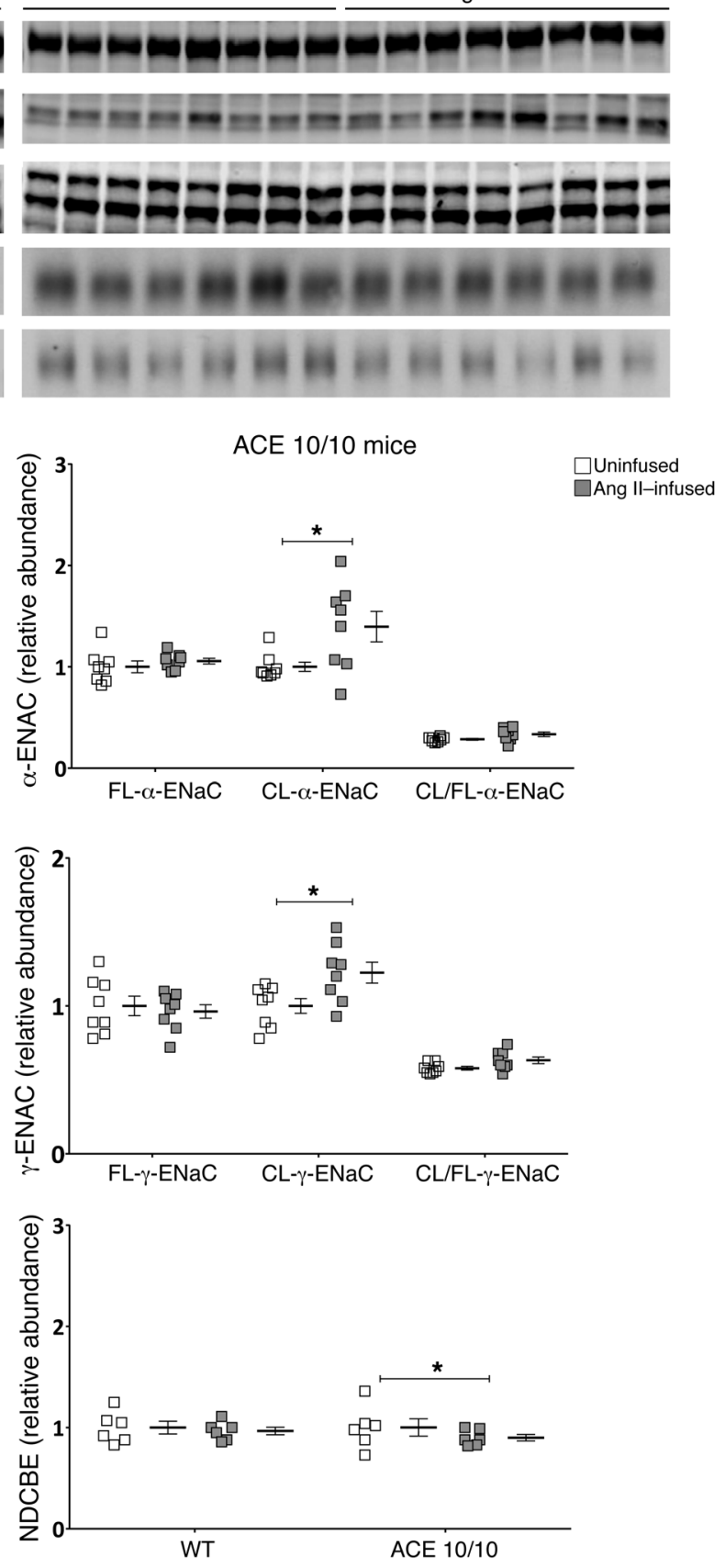

Figure 5

The absence of kidney ACE prevents changes in expression of $\alpha$ and $\gamma$ subunit of ENaC and pendrin induced by Ang II infusion. Transporter expression was analyzed in whole renal tissue homogenates extracted from uninfused (sham-operated) mice or hypertensive wild-type and ACE 10/10 mice after 2 weeks of Ang II infusion ( $400 \mathrm{ng} / \mathrm{kg} / \mathrm{min}$ via minipump). (A) Immunoblots were performed with a constant amount of protein per lane (Supplemental Table 1). (B) Relative abundance is displayed as individual measurements and mean \pm SEM. Since cleavage of $\alpha$ and $\gamma$ subunits is a marker for ENaC activation, the densities of both full-length (FL) and cleaved (CL) $\alpha$ - and $\gamma$-ENaC were calculated within each sample and as the resultant ratios of cleaved/full-length ENaC. $n=6-8$ per group. ${ }^{\star} P<0.05,{ }^{\star \star \star} P<0.001$.

kidneys from Ang II-infused wild-type mice (Supplemental Figure 10). In contrast, in Ang II-infused ACE 10/10 mice, the abundance of SPAK isoforms was unaltered and the phosphorylation levels of OSR1 and SPAK were unchanged.

\section{Discussion}

This study examines the precise role of intrarenal ACE in the response to hypertensive stimuli. Our initial experiments used 2 different lines of genetically modified mice: the ACE 10/10 model, 
A

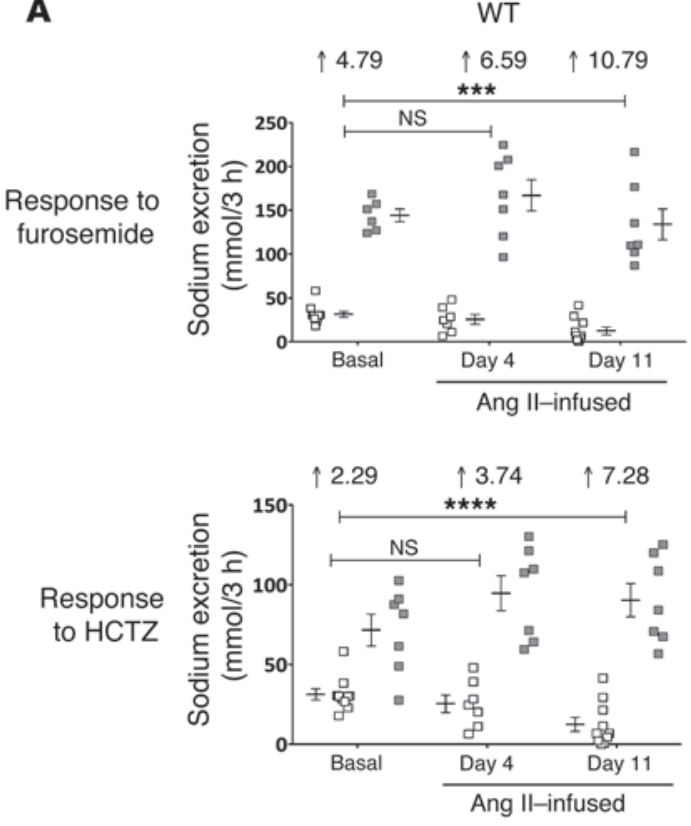

B

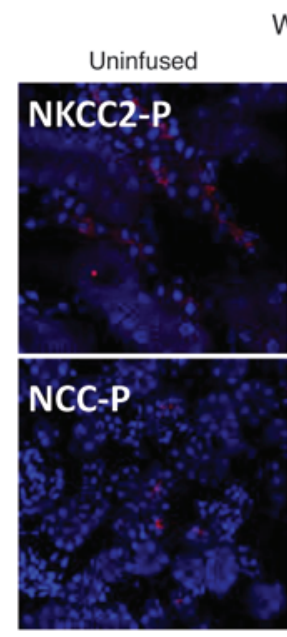

WT

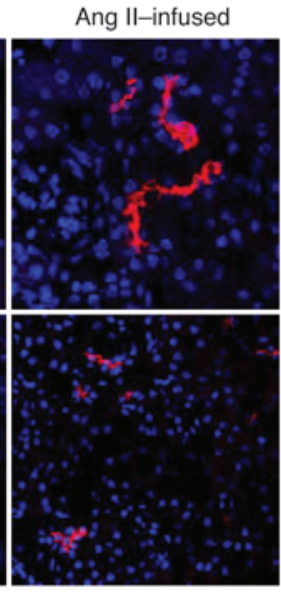

$\square$ Vehicle
$\square$ Diuretic

ACE $10 / 10$
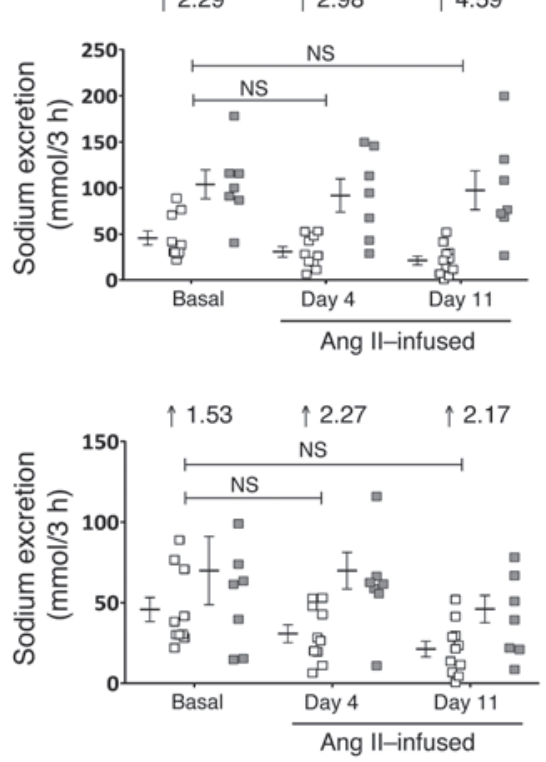

ACE 10/10

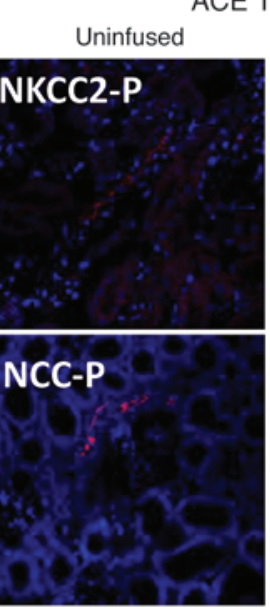

Ang II-infused

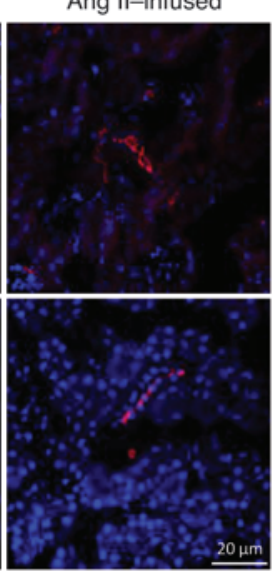

\section{Figure 6}

The absence of kidney ACE prevents the in vivo activation of NKCC2 and NCC induced by Ang II infusion, as determined by natriuretic responses to specific blockers and confocal microscopy. (A) Transport activity in wild-type and ACE 10/10 mice was assessed by measuring the natriuretic responses after a single i.p. bolus of furosemide (an NKCC2 blocker; $25 \mathrm{mg} / \mathrm{kg}$ ) or HCTZ (a NCC blocker; 25 mg/kg). Testing was performed before and after Ang II infusion (400 ng/kg/min). Numbers represent fold increase (arrows) over response in timematched mice injected with vehicle. $n=7-10 .{ }^{* * *} P<0.01,{ }^{* * \star *} P<0.0001$. Values represent individual measurements and mean \pm SEM. Basal natriuretic and diuretic response was significant in both wild-type $(P<0.0001$ for furosemide, $P<0.01$ for HCTZ) and ACE 10/10 mice $(P<0.01$ for furosemide, $P<0.05$ for HCTZ). (B) Immunofluorescence localization of NKCC2-P and NCC-P (red) in the thick ascending limb and distal tubules of kidneys of uninfused or Ang II-infused wild-type and ACE 10/10 mice. Uninfused and Ang II-infused samples were examined on the same slide with identical settings. Nuclei are stained blue with DAPI. Scale bar: $20 \mu \mathrm{m}$. an inbred line in which mice express ACE in myelomonocytic cells, and the ACE $3 / 3$ model, a line with mixed genetic background in which ACE is predominantly made by hepatocytes. In both models, plasma levels of ACE and Ang II and blood pressure levels are similar to those of control mice. Further, neither model shows any evidence of renal structural abnormalities $(8,9)$. Finally, and most importantly, both of these models express either no renal ACE (ACE 10/10 mice) or only minimal amounts of renal ACE (ACE $3 / 3$ mice). Thus, these mice with two separate and different patterns of Ace gene expression allowed us to study the role of renal ACE in the setting of normal kidney function and blood pressure.

Initially, hypertension in the 2 strains of mice was induced by 2 different protocols selected on the basis of their capacity to activate the intrarenal RAS $(10,11)$; blood pressure was investigated in response to a 2-week infusion of Ang II and in response to nitric oxide synthesis inhibition with L-NAME. In Ang IIinduced hypertension, plasma levels of Ang II are maintained at high levels. L-NAME-induced hypertension is associated with both low renin and low plasma Ang II levels. Strikingly, both mouse models responded to these 2 different forms of experimental hypertension with a substantially blunted hypertensive response (Figure 1). These data strongly suggest that removing ACE from the kidneys offers significant protection against experimental hypertension regardless of the systemic RAS status. Moreover, the protective effect extends beyond the specific genetic manipulation or strain background.

Next, we assessed the importance of local ACE in the renal responses to experimental hypertension. To this end, changes in the sodium and urine output of wild-type and ACE 10/10 mice were analyzed during Ang II infusion. This approach was selected for 2 reasons. First, the changes in kidney function of Ang IIinfused C57BL/6J mice are well characterized (26). Second, the constant infusion of the peptide eliminates potential differences in circulating Ang II between the wild-type and mutant mice. Indeed, with the exception of those recorded on day 1, plasma Ang II levels were similar in wild-type and mutant mice throughout the experiment. Ang II caused a transient reduction of sodium and urine output in wild-type mice that returned to preinfusion 
A
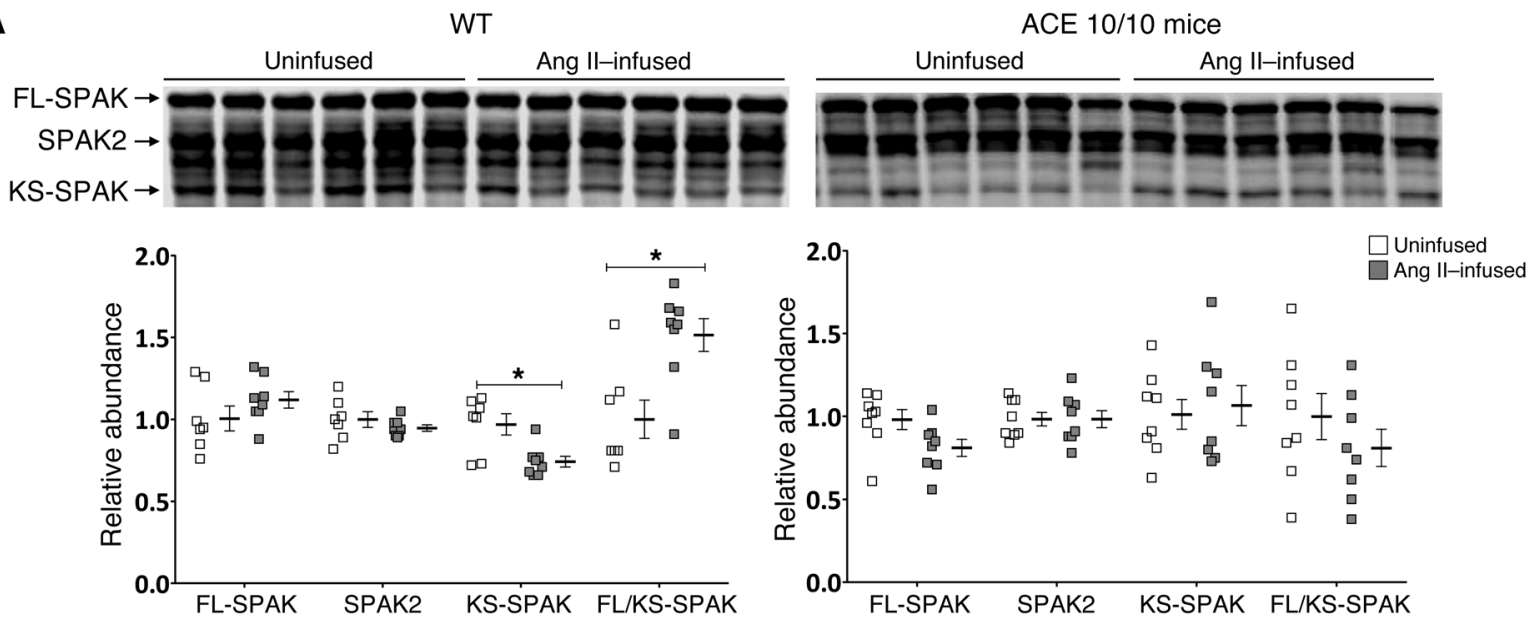

$\mathbf{B}$

WT
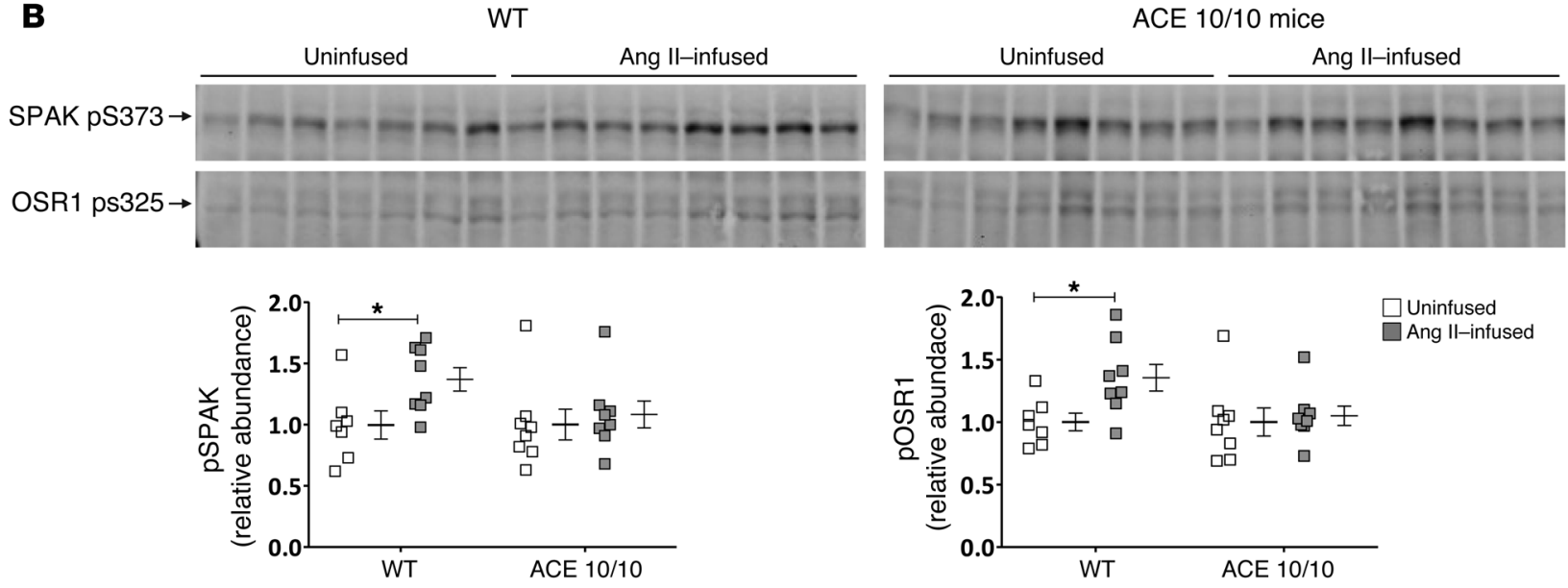

Figure 7

The absence of kidney ACE prevents the isoform shift of SPAK as well as SPAK and OSR1 phosphorylation induced by Ang II infusion. (A) SPAK isoform shift. Isoforms were identified using a combination of custom-made antibodies (Supplemental Figure 13) in whole renal tissue homogenates from wild-type and ACE 10/10 mice 2 weeks after sham operation (uninfused group) or after 2 weeks of Ang II infusion (400 ng/ $\mathrm{kg} / \mathrm{min}$ via minipump). Samples were run at a constant amount of protein per lane (Supplemental Table 1). Relative abundance of FL-SPAK, SPAK2, and KS-SPAK is displayed as are individual measurements and mean \pm SEM. Since KS-SPAK exerts a dominant-negative effect on SPAK kinase activity, the ratio of FL-SPAK to KS-SPAK (FL/KS-SPAK) provides a measure of SPAK activation. (B) Phosphorylation of SPAK and OSR1. Immunoblots of whole kidney homogenates were performed as described above. Relative abundance of phosphorylated SPAK and OSR1 (pSPAK and pOSR 1 ) is displayed as individual mice and mean \pm SEM. $n=6-8$ per group. ${ }^{*} P<0.05$.

levels after 48 to 72 hours (Figure 2). These changes were substantially blunted in the ACE 10/10 mice. After 3 days of Ang II infusion, sodium and urine output were similar in both groups. However, an examination of the MAP traces (Figure 1C) revealed a blood pressure increase in wild-type mice, concurrent with the return of sodium and urine output to baseline levels. In contrast, such an elevation was not observed in the ACE $10 / 10$ mice. These data are consistent with the kidney-fluid system hypothesis of Guyton (27). Specifically, Ang II infusion provoked a long-term increase of sodium reabsorption that raised blood pressure to a level requisite for the excretion of normal amounts of sodium and urine. Importantly, the absence of these changes in the ACE 10/10 mice implies that removal of kidney ACE prevents the effect of Ang II infusion that shifts the pressure-natriuresis relationship toward hypertension. The initial drop in sodium and urine excretion in wild-type mice may be explained by the higher plasma
Ang II during the first 24 hours in this group and its acute effects on renal hemodynamics, NHE3 activity, or both (28). It is unclear why plasma Ang II levels were initially higher in wild-type mice. Nevertheless, these acute responses should not distract from the observation that the steady-state urine and sodium output correction in the ACE 10/10 mice occurred at normal blood pressure levels, despite plasma Ang II levels similar to those of wild-type mice at day 3 and thereafter. Hence, the long-term protection against hypertension conferred by removal of kidney ACE was clearly independent from plasma Ang II levels.

One possible explanation for the protective phenotype against hypertension of mice lacking kidney ACE is reduced intrarenal Ang II synthesis. In support of this, the elevation of intrarenal Ang II content elicited by Ang II infusion was markedly attenuated in ACE 10/10 mice when compared with that in wild-type mice (Figure 2). While the idea of exogenous Ang II stimulating the renal 

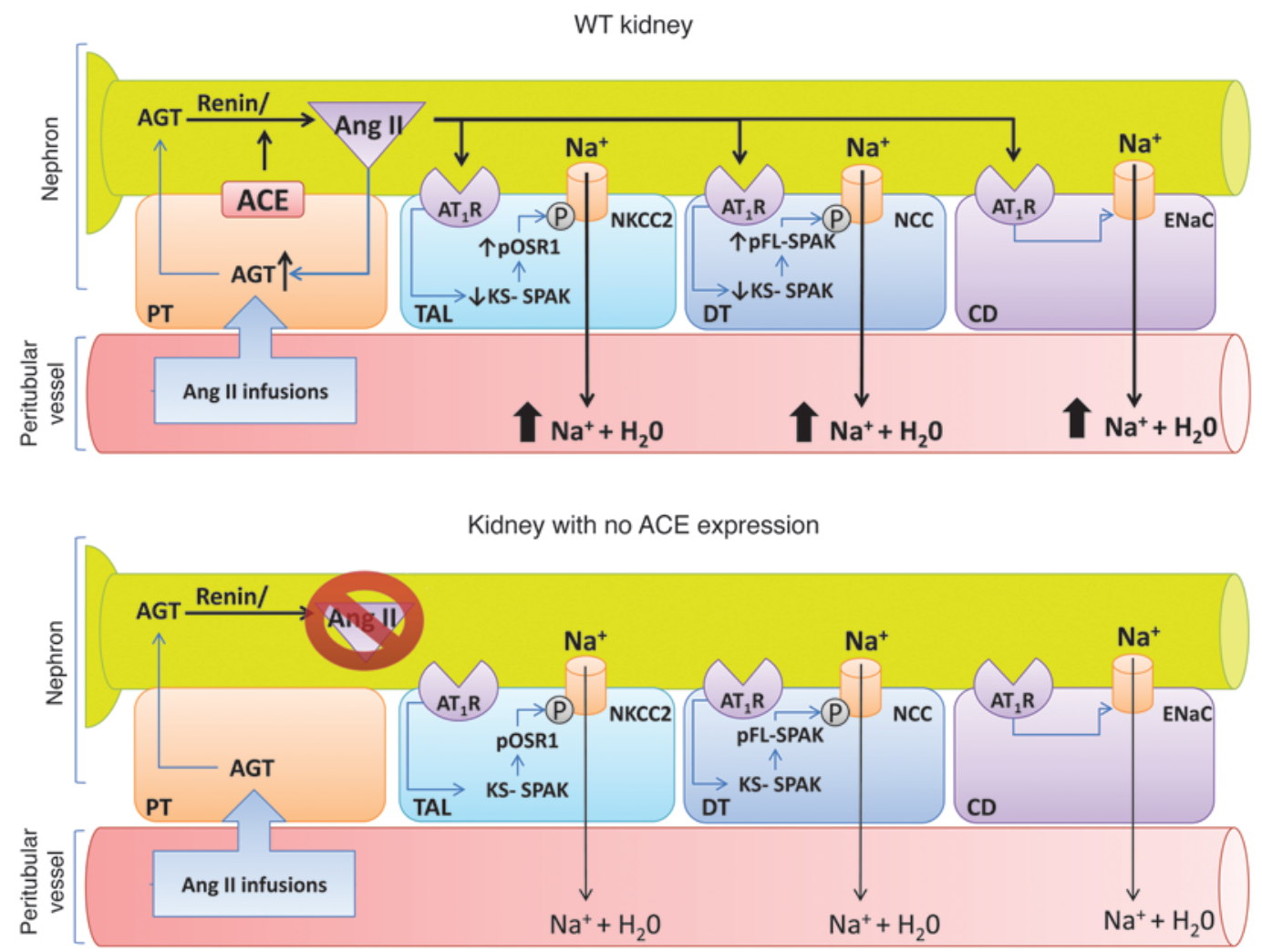

\section{Figure 8}

Hypothetical model for the actions of kidney ACE-derived Ang II synthesis in stimulating the activity of sodium transporters in the loop of Henle and distal nephron. Different conditions, including inflammation, reactive oxygen species, and Ang II, can act synergistically to induce angiotensinogen accumulation in proximal tubule cells, with its subsequent spillover into the tubular lumen and urine. After cleavage by renin and ACE, Ang II is released to trigger the activity of sodium transporters via AT ${ }_{1}$ receptors. These sequential events are hampered by the absence of kidney ACE. A nephron and the corresponding peritubular vessel are depicted in green and red, respectively. PT, proximal tubule; TAL, thick ascending limb; DT, distal tubule; CD, collecting duct.

synthesis of additional Ang II is not the prevailing view of the RAS, multiple reports suggest that the expression of several intrarenal RAS components, including angiotensinogen, ACE, tubular renin, and the $\mathrm{AT}_{1}$ receptor, is either augmented or sustained during Ang II-induced hypertension (11). The important role played by the enhanced activity of the intrarenal RAS during Ang II infusion is highlighted by previous reports. Experiments in rats infused with $\mathrm{Val}^{5}$-Ang II, an isoform of Ang II that can be separated from endogenous Ang II (Ile ${ }^{5}$-Ang II) by high-performance liquid chromatography, demonstrated that the chronic Val'5-Ang II (exogenous Ang II) infusion induces renal Ile ${ }^{5}$-Ang II (endogenous Ang II) synthesis (29). In another study, Ang II-infused mice became normotensive when endogenous Ang II production was reduced by ACE inhibition (15). It was also shown that Ang II causes hypertension primarily through stimulation of $\mathrm{AT}_{1}$ receptors in the kidneys (30). Finally, in our mouse model expressing ACE only in the kidneys and no other tissues, infusion of Ang I increased intrarenal Ang II content and induced hypertension, even though the mice exhibited renal structural abnormalities and low blood pressure (3).

Recently, it was shown that the liver is the main source of angiotensinogen in the kidneys under basal conditions (16). However, this observation does not explain the increases of angiotensinogen mRNA in the kidneys of Ang II-infused animals $(4,14,31)$. In our study, Ang II infusion increased liver angiotensinogen expression to a similar magnitude in wild-type and the ACE 10/10 mice. In contrast, kidney and urinary angiotensinogen increased only in wild-type mice in response to Ang II infusion (Figure 3). The failure to increase kidney and urinary angiotensinogen in the mutant mice is not a surprising result; it is known that renal Ang II exerts a powerful stimulation over angiotensinogen production and thus, by eliminating renal ACE and local Ang II synthesis, this stimulus is effectively hampered $(4,32)$. More importantly, the differential regulation of kidney angiotensinogen in the ACE 10/10 mice supports a renal-specific effect of the genetic manipulation and a cause-effect relationship between the absence of kidney ACE and the protective phenotype. This is substantiated by evidence demonstrating that most urinary angiotensinogen is produced by the kidneys and earlier publications showing that proximal tubule angiotensinogen levels regulate blood pressure (4-6, 33, 34). In this context, the increasing accumulation of angiotensinogen and the persistence of renin activity, due to tubular renin upregulation (35), in kidneys from Ang II-infused animals help explain why local ACE plays such an important role in regulating the local pool of Ang II in this condition.

In addition to Ang I, ACE cleaves other substrates, including bradykinin, neurotensin, AcSDKP, enkephalins, and others (36). With 
the exception of bradykinin, none of the other known substrates of ACE appear to be good candidates to explain our data. Bradykinin accumulates in the absence of ACE or during its inhibition (7) and causes vasodilation and natriuresis mostly by $\mathrm{B}_{2}$ receptor activation of eNOS. However, the importance of bradykinin in experimental hypertension is not clear $(36,37)$. We find that ACE 10/10 mice, lacking renal ACE, display a reduced hypertensive response to long-term treatment with L-NAME. This suggests that their protective phenotype is independent of the nitric oxide status and, by extension, perhaps bradykinin. Finally, the hemodynamic contributions of other ACE substrates, including bradykinin, are dwarfed by the potent effects of Ang II to regulate kidney function.

Besides the kidney, ACE is abundantly expressed by endothelium; lesser amounts are found in the gut, certain immune cells, and the brain $(35,38)$. Therefore, the possibility exists that the absence of endothelial ACE or the ACE from some other organ contributes to the responses observed in this study. In the case of endothelial ACE, the two experimental conditions used in this study, in particular Ang II infusion, cause strong plasma renin suppression (14). This effectively reduces plasma Ang I formation and minimizes any potential contribution of endothelial ACE. It is also conceivable that the ectopic expression of ACE in our models affects our results. In particular, ACE is made in large amounts in the livers of the ACE $3 / 3$ mice. Although we did not detect liver morphological or functional abnormalities, it is possible that the liver ACE modulates hepatorenal reflexes in the ACE $3 / 3$ mice. While work by others highlights the importance of these reflexes in the regulation of postprandial natriuresis, such a mechanism seems to be less important for longterm sodium homeostasis (39).

Based on our preceding comments, we conclude that the most logical explanation for our findings is that removing kidney ACE impairs intrarenal Ang II formation, thereby reducing renal sodium and fluid reabsorption and the accompanying development of hypertension. It is noteworthy that most of our observations were made during constant infusion of exogenous Ang II, suggesting that the effects of intrarenal Ang II on renal sodium transport regulation are nonredundant with systemic Ang II. Using a similar argument, we attribute the residual increases in blood pressure observed in mice with no kidney ACE to the actions of exogenous Ang II in the kidneys and other organs, including the vasculature and the central nervous system. The potential impact of removing kidney ACE on local ACE2 expression and Ang-(1-7) production is excluded from our analysis. In many instances, the renal actions of Ang-(1-7) counteract those of Ang II (40). Therefore, the possibility exists that some of the protection observed in the mutant mice is due to the unopposed actions of the Ang-(1-7)/ Mas receptor axis. In addition, we did not analyze the responses of the mutant mice to changes in endogenous Ang II formation induced by variations in dietary sodium.

A goal of our study was to identify downstream targets (direct or indirect) of intrarenal Ang II. To this end, we profiled the expression of sodium transporters in kidneys from normal mice and ACE 10/10 mice lacking kidney ACE. At baseline, the transporter profiles were indistinguishable. However, Ang II infusion triggered an array of responses in wild-type mice consistent with increased sodium transport in the loop of Henle and the distal nephron: increased phosphorylation of loop of Henle NKCC2, increased abundance and phosphorylation of the distal convoluted tubule NCC, increased cleavage of $\mathrm{ENaC} \alpha$ and $\gamma$ subunits, and increased abundance of the intercalated cell anion transporter pendrin (Figures 4 and 5). The initial changes were detected as early as 4 days after infusion and were most evident at days 10 and 14 . In contrast, most of these responses were eliminated in mice lacking kidney ACE, despite the fact that they were also infused with Ang II. The proteolytic cleavage of ENaC subunits, a marker of $\mathrm{ENaC}$ activation, was the only response evident in Ang II-infused ACE 10/10 mice, suggesting that this response is mediated by systemic Ang II or aldosterone or that it is very sensitive to low levels of tubular Ang II. The lack of stimulation of the other transporters is consistent with the enhanced natriuresis and blunted hypertension of the ACE 10/10 mice. In summary, our findings indicate that the long-term pressure-natriuresis shift observed during Ang II infusion in wild-type mice is associated with a stimulatory effect on transporter abundance and/or activity in the loop of Henle and more distal segments. Even more importantly, these effects required intrarenal Ang II synthesis, demonstrating for what we believe to be the first time a fundamental role of local Ang II as a master regulator of sodium transport along the nephron.

Previous studies have explored Ang II regulation of NKCC2, NCC (41-44), $\operatorname{ENaC}(45,46)$, and pendrin $(47,48)$, yet a major finding of this study is the unique role of intrarenal Ang II in triggering their activation. This observation adds a novel element to the multiple pathways converging in the distal nephron to maintain sodium and fluid homeostasis $(49,50)$. The remarkable changes in NKCC2 and NCC phosphorylation elicited by intrarenal Ang II prompted us to analyze their regulation in more detail. First, our semiquantitative analysis by confocal microscopy confirmed the immunoblot findings (Figure 6). In addition, diuretic tests demonstrated physiologic correlates to the increased transporter activity in wild-type mice but not in mice with reduced renal ACE expression (Figure 6 and Supplemental Figure 10). Recent studies indicate that NKCC2 phosphorylation is mainly driven by OSR1 while NCC phosphorylation is driven by FL-SPAK (51-53). SPAK was first identified by whole-genome association studies as a susceptibility gene for hypertension (54). We examined whether there was evidence of SPAK or OSR1 regulation by renal ACE. While we did not observe any change in OSR1 abundance, in Ang II-infused wild-type mice, we detected changes in the isoform expression of SPAK as early as 4 days after infusion (Figure 7 and Supplemental Figure 9). We specifically observed a sustained reduction of KS-SPAK expression and an early increased in FL-SPAK abundance. These changes are reminiscent of those evoked by extracellular volume depletion, attributed to elevated Ang II (51). According to McCormick et al., a KS-SPAK reduction releases its inhibitory influence on both OSR1 and FL-SPAK, allowing these kinases to phosphorylate NKCC2 and NCC, respectively $(51,53)$. Consistent with this, we observed increased phosphorylation of SPAK and OSR1 only in wild-type mouse kidneys (Figure 7). Thus, we conclude that the reduction in KS-SPAK, as well as the increased phosphorylation of SPAK and OSR1, are dependent on the presence of intrarenal ACE during Ang II infusion, presumably due to the intrarenal Ang II synthesis.

Our final consideration with regards to ACE is the locus for Ang II synthesis within the kidney. ACE is expressed in at least 5 different renal cell types. However, most of the renal ACE is located in the apical side of straight proximal tubules (38). Accordingly, we provided a hypothetical model (Figure 8 ) in which we surmise that in conditions in which the intrarenal RAS is activated (for instance, 
during chronic Ang II infusion) the activity of proximal tubule ACE leads to an increased luminal Ang II synthesis and further angiotensinogen accumulation. The newly formed peptide then stimulates, directly or indirectly via regulatory kinases, sodium and chloride transporters located downstream in the loop of Henle and the distal nephron. The resulting increased sodium avidity of the kidneys elicits a rightward shift on the pressure-natriuresis relationship and the hypertension.

In conclusion, the results of this study demonstrate that induction of kidney ACE-derived Ang II formation plays an important role in the generation of hypertension. We introduce a paradigm in which the activity of kidney ACE, and the resultant local Ang II synthesis, is required to increase the abundance, phosphorylation, and/or processing of NKCC2, NCC, $\mathrm{ENaC}$, and pendrin in the loop of Henle and the distal nephron, thereby increasing sodium and fluid reabsorption and blood pressure. Furthermore, our findings suggest that reducing renal ACE activity would be an effective means to manipulate this pathway, offering a new area for potential organ-specific intervention.

\section{Methods}

Animals. The generation of ACE 10/10 and ACE 3/3 mice has been described elsewhere $(8,9)$. Targeted homologous recombination was used to place Ace gene expression under the control of the c-fms promoter (ACE 10/10 mice) or the albumin promoter (ACE $3 / 3$ mice). These changes resulted in restricted expression of ACE to myelomonocytic cells and the liver, respectively. ACE 10/10 mice had been backcrossed to a pure C57BL/6J background. Although the genetic background of ACE $3 / 3$ mice is mixed between $129 \mathrm{j}$ and $\mathrm{C} 57 \mathrm{BL} / 6 \mathrm{~J}$, these mice were selected to harbor only one renin gene $(\operatorname{Ren} 1 c)$, a model more similar to humans. The experimental protocols were conducted in 8- to 12-week-old male mice of either strain and wild-type mice of the corresponding genetic background.

Experimental hypertension and blood pressure monitoring. Hypertension was induced by subcutaneous infusion of Ang II ( $400 \mathrm{ng} / \mathrm{kg} / \mathrm{min}$ ) via osmotic minipump (Alzet 1002, Durect Corporation) or by L-NAME treatment $(5 \mathrm{mg} / 10 \mathrm{ml}$ in the drinking water). Blood pressure was followed by tailcuff plethysmography every 3 days using a Visitech BP2000 system (Visitech Systems Inc.) or by telemetry. For the latter, mice were anesthetized with isoflurane, and a catheter connected to a radiotelemetry device (model PA-C10; Data Sciences International) was inserted in the left carotid artery. This allowed monitoring of heart rate and MAP in unrestrained conditions. After a 14-day recovery phase, baseline levels were established briefly before Ang II infusion. Data were collected, stored, and analyzed using Dataquest A.R.T. 4.0 software (Data Sciences International) (15).

Metabolic studies and diuretics challenge. For studies requiring urine sampling, mice were individually housed in metabolic cages. For balance experiments, mice were fed a gelled $0.25 \% \mathrm{NaCl}$ diet that contained all nutrients and water (Nutra-gel; Bio-Serv). Following 3 days of baseline collections, sham operation or minipump implantation was performed. Immediately after surgery, mice were returned to the metabolic cages for 3 additional days and again at days 11 and 12 after minipump implantation. For the diuretics challenge, after voiding the bladder by gently massaging the lower abdomen, mice were injected with a single bolus of a diuretic (HCTZ or furosemide, $25 \mathrm{mg} / \mathrm{kg}$ i.p.) or vehicle $(0.9 \% \mathrm{NaCl}$ solution $)$ and placed in metabolic cages, and urine samples were collected for 3 hours. Free access to food and water was allowed at all times. Diuretic treatment occurred once 48 hours before minipump implantation and at days 4 and 11 after implantation. The following parameters were monitored: water and food ingestion and urine and sodium output. Sodium concentration was measured by flame photometry.
Transporter profiling and intrarenal RAS analysis. Whole mouse kidneys, flash frozen after removal, were thawed and homogenized, as described in detail elsewhere (55). Protein concentration was determined by BCA, and samples were denatured in SDS-PAGE sample buffer (20 minutes, $60^{\circ} \mathrm{C}$ ), resolved by SDS-PAGE (56), transferred to polyvinylidene difluoride membranes (Millipore Immobilon-FL), blocked (Li-COR blocking buffer or bløk-FL, Millipore), and then probed with specific antibodies, as detailed in Supplemental Table 1. To verify uniform loading, loading gels were run (5 $\mu \mathrm{g}$ per lane) and stained with Coomassie blue, and random bands were quantified. Linearity of signal intensity was established by loading 0.5 volumes of each sample on each gel to verify doubling of signal intensity. Signals on immunoblots were detected and quantitated with Odyssey Infrared Imaging System (Li-COR) and accompanying software. Values were normalized to the mean intensity measured in the wild-type or uninfused groups defined as 1.0. All comparisons were performed between samples run on the same blot/membrane.

Determinations of ANG peptides. Trunk blood was collected by conscious decapitation into chilled tubes containing a protease inhibitor cocktail (14). For intrarenal Ang II, the right kidneys were homogenized in methanol immediately after extraction. Later, plasma and kidneys samples were processed as described elsewhere (14).

Indirect immunofluorescence. To examine NKCC2-P and NCC-P regulation in situ, we performed immunofluorescence microscopy experiments. Kidneys were perfusion-fixed via the heart, cryoprotected in $30 \%$ sucrose PBS, and then embedded and frozen in liquid nitrogen. Cryosections were cut, and sections of both uninfused and Ang II-infused mice were collected on the same charged object glass slide, side by side, to assure comparable treatments. Samples were subjected to antigen retrieval and incubated with primary and secondary antibodies (Supplemental Table 1), as described in detail elsewhere (28). Samples were viewed with a Leica DMI 6000 inverted microscope and imaged using a Leica TCS SP5 confocal fluorescence imaging system powered by a Chameleon Ultra-II MP laser (Coherent Inc.) and visible RGB lasers (Leica Microsystems). Fluorescence excitation and detector settings were the same for imaging renal sections of uninfused and Ang II-infused mice.

Immunohistochemistry. Four-micron sections were cut from paraffinembedded blocks onto positively charged slides. Deparaffinization and pretreatment of the sections were done in the Dako PT Link Module using Dako Low pH buffer. Immunohistochemical staining was done on the Dako Autostainer. For morphological analysis, the sections were counterstained with H\&E and then coverslipped after dehydration through alcohol and xylene. For macrophage staining, the sections were blocked with $3 \% \mathrm{H}_{2} \mathrm{O}_{2}$ and incubated with rat anti-mouse $\mathrm{F} 4 / 80$ antibody from $\mathrm{AbD}$ Serotec (1:100 dilution for 45 minutes). A Rat-on-Mouse HRP-Polymer Kit from Biocare and DAB Kit from Dako were used for detecting antibody binding. The sections were counterstained with hematoxylin and then coverslipped after dehydration through alcohol and xylene. Stained slides were scanned with a Leica SCN400 Slide Scanner. Digital images of whole kidney sections were saved for analysis. Evaluation was performed on 10 random fields at $\times 200$ magnification, where each field represents $0.25 \mathrm{~mm}^{2}$, resulting in a total explored area of $2.5 \mathrm{~mm}^{2}$. The images were analyzed with ImageJ (version 1.46r; NIH), and averaged results were expressed as F4/80-positive cells per field.

Statistics. All data are presented as individual measurements along with mean \pm SEM. Two-way ANOVA with Bonferroni's post-test was used when analyzing changes in data collected over time. One-way ANOVA and 2-tailed unpaired Student's $t$ test were used to analyze differences between controls and hypertensive mice within the same genotype or to assess the differences between wild-type and mutant mice when appropriate. All statistical tests were calculated using GraphPad Prism 5.00 (GraphPad Software). A value of $P<0.05$ was regarded as significant. 
Study approval. The experiments described in this manuscript were conducted with the approval of the Cedars-Sinai Institutional Animal Care and Use Committee.

\section{Acknowledgments}

This study was supported by NIH grants R00DK083455 (to R.A. Gonzalez-Villalobos), P20RR017659 and HL26371 (to L.G. Navar), R01HL110353 (to K.E. Bernstein), HL088000 (to S. Fuchs), GM074771 (to E. Delpire), and DK083785 (to A.A. McDonough) and a grant from the University Kidney Research Organization (to A.A. McDonough, R.A. Gonzalez-Villalobos, and J. Peti-Peterdi). D. Eladari and N. Picard are funded by grant "HYPERCLO" ANR BLANC 2010-R10164DD from l'Agence Nationale de la Recherche
(ANR). The authors thank Gail Thomas for allowing them access to her telemetry equipment, and Fai Chung for her assistance in immunohistochemistry studies.

Received for publication July 30, 2012, and accepted in revised form February 21, 2013.

Address correspondence to: Romer A. Gonzalez-Villalobos, Assistant Professor of Biomedical Sciences and Pathology and Laboratory Medicine, Cedars-Sinai Medical Center, $8700 \mathrm{Bev}-$ erly Blvd., Davis Bldg., Rm. 2092, Los Angeles, California 90048, USA. Phone: 310.423.0551; Fax: 310.423.7331; E-mail: romer. gonzalez@csmc.edu.
1. Atlas SA. The renin-angiotensin aldosterone system: pathophysiological role and pharmacologic inhibition. J Manag Care Pharm. 2007;13(8 suppl B):9-20.

2. Alderman MH, Cohen HW, Sealey JE, Laragh JH. Plasma renin activity levels in hypertensive persons: their wide range and lack of suppression in diabetic and in most elderly patients. Am J Hypertens. 2004;17(1):1-7.

3. Gonzalez-Villalobos RA, et al. Intrarenal angiotensin-converting enzyme induces hypertension in response to angiotensin I infusion. J Am Soc Nephrol. 2011;22(3):449-459.

4. Kobori H, et al. Kidney-specific enhancement of ANG II stimulates endogenous intrarenal angiotensinogen in gene-targeted mice. Am J Physiol Renal Physiol. 2007;293(3):F938-F945.

5. Lavoie JL, Lake-Bruse KD, Sigmund CD. Increased blood pressure in transgenic mice expressing both human renin and angiotensinogen in the renal proximal tubule. Am J Physiol Renal Physiol. 2004; 286(5):F965-F971.

6. Sachetelli S, et al. RAS blockade decreases blood pressure and proteinuria in transgenic mice overexpressing rat angiotensinogen gene in the kidney. Kidney Int. 2006;69(6):1016-1023.

7. Campbell DJ, et al. Effect of reduced angiotensinconverting enzyme gene expression and angiotensin-converting enzyme inhibition on angiotensin and bradykinin peptide levels in mice. Hypertension. 2004;43(4):854-859.

8. Shen XZ, et al. Mice with enhanced macrophage angiotensin-converting enzyme are resistant to melanoma. Am J Pathol. 2007;170(6):2122-2134.

9. Cole J, Quach DL, Sundaram K, Corvol P, Capecchi MR, Bernstein KE. Mice lacking endothelial angiotensin-converting enzyme have a normal blood pressure. Circ Res. 2002;90(1):87-92.

10. Graciano ML, et al. Intrarenal Renin-Angiotensin system is upregulated in experimental model of progressive renal disease induced by chronic inhibition of nitric oxide synthesis. J Am Soc Nephrol. 2004;15(7):1805-1815.

11. Navar LG, Kobori H, Prieto MC, Gonzalez-Villalobos RA. Intratubular renin-angiotensin system in hypertension. Hypertension. 2011;57(3):355-362.

12. Sandberg K, Ji H. Sex differences in primary hypertension. Biol Sex Differ. 2012;3(1):7.

13. Pollock DM, Polakowski JS, Divish BJ, Opgenorth TJ. Angiotensin blockade reverses hypertension during long-term nitric oxide synthase inhibition. Hypertension. 1993;21(5):660-666.

14. Gonzalez-Villalobos RA, et al. Intrarenal angiotensin II and angiotensinogen augmentation in chronic angiotensin II-infused mice. Am J Physiol Renal Physiol. 2008;295(3):F772-F779.

15. Gonzalez-Villalobos RA, et al. Angiotensin-converting enzyme-derived angiotensin II formation during angiotensin II-induced hypertension. Hypertension. 2009;53(2):351-355.

16. Matsusaka T, et al. Liver angiotensinogen is the primary source of renal angiotensin II. J Am Soc Nephrol. 2012;23(7):1181-1189.

17. Hall JE, Guyton AC, Coleman TG, Mizelle HL, Woods LL. Regulation of arterial pressure: role of pressure natriuresis and diuresis. Fed Proc. 1986;45(13):2897-2903.

18. Mitchell KD, Braam B, Navar LG. Hypertensinogenic mechanisms mediated by renal actions of renin-angiotensin system. Hypertension. 1992; 19(1 suppl):I18-I27.

19. Masilamani S, Kim GH, Mitchell C, Wade JB, Knepper MA. Aldosterone-mediated regulation of $\mathrm{ENaC}$ alpha, beta, and gamma subunit proteins in rat kidney. J Clin Invest. 1999;104(7):R19-R23.

20. Ares GR, Caceres PS, Ortiz PA. Molecular regulation of NKCC2 in the thick ascending limb. AmJ Physiol Renal Physiol. 2011;301(6):F1143-F1159.

21. Lee DH, et al. Effects of ACE inhibition and AngII stimulation on renal $\mathrm{Na}-\mathrm{Cl}$ cotransporter distribution, phosphorylation and membrane complex properties. Am J Physiol Cell Physiol. 2013; 304(2):C147-C163.

22. Moriguchi T, et al. WNK1 regulates phosphorylation of cation-chloride-coupled cotransporters via the STE20-related kinases, SPAK and OSR1.J Biol Chem. 2005;280(52):42685-42693.

23. Ponce-Coria J, et al. Regulation of NKCC2 by a chloride-sensing mechanism involving the WNK3 and SPAK kinases. Proc Natl Acad Sci U S A. 2008; 105(24):8458-8463.

24. Richardson C, et al. Activation of the thiazidesensitive $\mathrm{Na}^{+}-\mathrm{Cl}^{-}$cotransporter by the WNK-regulated kinases SPAK and OSR1. J Cell Sci. 2008; 121(pt 5):675-684.

25. Piechotta K, Garbarini N, England R, Delpire E. Characterization of the interaction of the stress kinase SPAK with the $\mathrm{Na}^{+}-\mathrm{K}^{+}-2 \mathrm{Cl}^{-}$cotransporter in the nervous system: evidence for a scaffolding role of the kinase. J Biol Chem. 2003;278(52):52848-52856.

26. Zhao D, Seth DM, Navar LG. Enhanced distal nephron sodium reabsorption in chronic angiotensin II-infused mice. Hypertension. 2009;54(1):120-126.

27. Guyton AC. Blood pressure control--special role of the kidneys and body fluids. Science. 1991; 252(5014):1813-1816.

28. Riquier-Brison AD, Leong PK, Pihakaski-Maunsbach K, McDonough AA. Angiotensin II stimulates trafficking of NHE3, $\mathrm{NaPi} 2$, and associated proteins into the proximal tubule microvilli. Am J Physiol Renal Physiol. 2010;298(1):F177-F186.

29. Shao W, Seth DM, Navar LG. Augmenation of endogenous Angiotensin II levels in Val5-Ang II infused rats. JIM. 2008;56:344-492.

30. Crowley SD, et al. Angiotensin II causes hypertension and cardiac hypertrophy through its receptors in the kidney. Proc Natl Acad Sci U S A. 2006; 103(47):17985-17990.

31. Kobori H, Harrison-Bernard LM, Navar LG. Enhancement of angiotensinogen expression in angiotensin II-dependent hypertension. Hyperten- sion. 2001;37(5):1329-1335.

32. Modrall JG, et al. Depletion of tissue angiotensinconverting enzyme differentially influences the intrarenal and urinary expression of angiotensin peptides. Hypertension. 2004;43(4):849-853.

33. Nakano D, et al. Multiphoton imaging of the glomerular permeability of angiotensinogen. J Am Soc Nephrol. 2012;23(11):1847-1856.

34. Ying J, et al. Overexpression of mouse angiotensinogen in renal proximal tubule causes salt-sensitive hypertension in mice. Am J Hypertens. 2012; 25(6):684-689.

35. Gonzalez-Villalobos RA, et al. Intrarenal mouse renin-angiotensin system during ANG II-induced hypertension and ACE inhibition. Am J Physiol Renal Physiol. 2010;298(1):F150-F157.

36. Shen XZ, et al. Nontraditional roles of Angiotensinconverting enzyme. Hypertension. 2012;59(4):763-768.

37. Duka I, Kintsurashvili E, Gavras I, Johns C, Bresnahan $M$, Gavras H. Vasoactive potential of the $b(1)$ bradykinin receptor in normotension and hypertension. Circ Res. 2001;88(3):275-281.

38. Alhenc-Gelas F, Baussant T, Hubert C, Soubrier F, Corvol $\mathrm{P}$. The angiotensin converting enzyme in the kidney. J Hypertens Suppl. 1989;7(7):S9-S13.

39. Morita H, Abe C. Negative feedforward control of body fluid homeostasis by hepatorenal reflex. Hypertens Res. 2011;34(8):895-905.

40. Ferrario CM, Varagic J. The ANG-(1-7)/ACE2/mas axis in the regulation of nephron function. Am J Physiol Renal Physiol. 2010;298(6):F1297-F1305.

41. San-Cristobal P, et al. Angiotensin II signaling increases activity of the renal $\mathrm{Na}-\mathrm{Cl}$ cotransporter through a WNK4-SPAK-dependent pathway. Proc Natl Acad Sci U S A. 2009;106(11):4384-4389.

42. van der Lubbe N, et al. Angiotensin II induces phosphorylation of the thiazide-sensitive sodium chloride cotransporter independent of aldosterone. Kidney Int. 2011;79(1):66-76

43. Gurley SB, et al. AT1A angiotensin receptors in the renal proximal tubule regulate blood pressure. Cell Metab. 2011;13(4):469-475.

44. Brooks HL, Allred AJ, Beutler KT, Coffman TM, Knepper MA. Targeted proteomic profiling of renal $\mathrm{Na}(+)$ transporter and channel abundances in angiotensin II type 1a receptor knockout mice. Hypertension. 2002;39(2 pt 2):470-473.

45. Mamenko M, Zaika O, Ilatovskaya DV, Staruschenko A, Pochynyuk O. Angiotensin II increases activity of the epithelial $\mathrm{Na}+$ channel $(\mathrm{ENaC})$ in distal nephron additively to aldosterone. J Biol Chem. 2012;287(1):660-671.

46. Peti-Peterdi J, Warnock DG, Bell PD. Angiotensin II directly stimulates ENaC activity in the cortical collecting duct via AT(1) receptors. J Am Soc Nephrol. 2002;13(5):1131-1135.

47. Verlander JW, et al. Angiotensin II acts through the angiotensin 1a receptor to upregulate pendrin. Am J Physiol Renal Physiol. 2011;301(6):F1314-F1325.

48. Pech V, Kim YH, Weinstein AM, Everett LA, Pham 
TD, Wall SM. Angiotensin II increases chloride absorption in the cortical collecting duct in mice through a pendrin-dependent mechanism. Am J Physiol Renal Physiol. 2007;292(3):F914-F920.

49. McCormick JA, Ellison DH. The WNKs: atypical protein kinases with pleiotropic actions. Physiol Rev. 2011;91(1):177-219.

50. Soundararajan R, Pearce D, Hughey RP, Kleyman TR. Role of epithelial sodium channels and their regulators in hypertension. J Biol Chem. 2010; 285(40):30363-30369.
51. McCormick JA, et al. A SPAK isoform switch modulates renal salt transport and blood pressure. Cell Metab. 2011;14(3):352-364.

52. Yang SS, et al. SPAK-knockout mice manifest Gitelman syndrome and impaired vasoconstriction. J Am Soc Nephrol. 2010;21(11):1868-1877.

53. $\mathrm{Lin} \mathrm{SH}$, et al. Impaired phosphorylation of $\mathrm{Na}(+)$ $\mathrm{K}(+)-2 \mathrm{Cl}(-)$ cotransporter by oxidative stress-responsive kinase- 1 deficiency manifests hypotension and Bartter-like syndrome. Proc Natl Acad Sci US A. 2011;108(42):17538-17543.
54. Liu X, et al. Genome-wide association study identifies candidate genes for Parkinson's disease in an Ashkenazi Jewish population. BMC Med Genet. 2011; 12:104.

55. Nguyen MT, et al. Effects of $\mathrm{K}^{+}$-deficient diets with and without $\mathrm{NaCl}$ supplementation on $\mathrm{Na}^{+}, \mathrm{K}^{+}$, and $\mathrm{H}_{2} \mathrm{O}$ transporters' abundance along the nephron. Am J Physiol Renal Physiol. 2012;303(1):F92-F104.

56. Laemmli UK. Cleavage of structural proteins during the assembly of the head of bacteriophage T4. Nature. 1970;227(5259):680-685. 